\title{
Deporte extracurricular: examinando el compromiso en relación con variables socioeducativas Extracurricular sports: examining commitment to socioeducational variables
}

Paula Francisca Ortiz Marholz*, Luis Javier Chirosa Ríos*, Ignacio Martín Tamayo*, Rafael Enrique Reigal Garrido*,Alejandro

\author{
García Mas**
}

*Universidad de Granada (España), **Universidad de las Islas Baleares (España)

Resumen. El presente estudio tuvo como objetivo identificar las diferencias en el compromiso deportivo entre centros educacionales (municipales, particular subvencionado, particulares) y escuelas de fútbol. Los participantes fueron 913 jugadores de fútbol entre 10 y 14 años (M: 12.2 DT: 1.3 ). Los instrumentos utilizados fueron las versiones en español del Sport Commitment Questionnaire - SCQ-e - y un cuestionario sociodemográfico. A partir de los resultados y análisis de significación, se pudo apreciar la existencia de diferencias en los factores potenciadores del compromiso donde los establecimientos públicos o municipales mostraron menores índices que los otros grupos. En esta línea, en aquellos factores que se asocian negativamente al compromiso, este tipo de establecimiento educacional mostró mayores índices. Estos resultados permiten reflexionar a acerca de la complejidad de los contextos que implican los programas impartidos, los profesionales a cargo y los objetivos que se persiguen. Esto permite proponer intervenciones considerando la realidad socioeducativa y las consecuencias que pueden generar.

Palabras clave. Futbolistas Jóvenes, Centros Educacionales, Modelo de Compromiso Deportivo, Actividades Alternativas.

Abstract. The present study aimed to identify possible differences in sport commitment between types of institutions, either educational (municipal, subsidized private, private) or football programs. Participants were 913 soccer players between 10 and 14 years (M: 12.2 SD: 1.3). The instruments used were the Spanish versions of the Sport Commitment Questionnaire - SCQ-e - and a sociodemographic questionnaire. From the results and analysis of significance, it was observed that there are differences in the factors enhancing commitment where public or municipal schools had lower rates than other groups. Likewise, regarding those factors that are negatively associated with commitment, this type of educational institution showed higher rates. These results allow to reflect about the programmes delivered and propose interventions considering the socio-educational reality and the consequences that may result.

Keywords. Young football players, Educational Centers, Sport Commitment Model, Alternative Activities.

\section{Introducción}

La mayoría de los niños y adolescentes realizan alguna práctica deportiva de forma regular, ya sea en el marco curricular o como actividad extraescolar, es así que podemos dar cuenta que existe un entorno importante de considerar en donde los niños y jóvenes se desenvuelven día a día, el contexto socio-educativo (García Ferrando, 2006; Rouling y Bangerter, 2013). Este contexto se distingue en cuanto a los recursos con los que se desarrolla y en las posibilidades de contar con infraestructura acorde a las actividades que ofrece. La literatura ha encontrado que la realización de actividad deportiva extraescolar es un espacio importante de investigar en cuanto es libremente elegida por los niños y jóvenes y puede crear vínculos de compromiso (Leo, Sánchez-Miguel, Sánchez-Oliva, Amado y García-Calvo, 2011).

El deporte y el ejercicio ofrecen oportunidades para que los niños y jóvenes establezcan su identidad y autopercepción y su relevancia ha sido reportada por varios investigadores y teóricos (Anderson y Cychosz, 1995; Brettschneider y Heim, 1997; Cachán, 2011; Curry, 1993; Curry y Weaner, 1987; Greendorfer, 2002; Theodorakis, 1994). La práctica deportiva es vista entonces, como un apoyo para el desarrollo personal, emocional y físico, cumpliendo un importante rol en la salud y a pesar que los niños deben participar en horas de educación física como requisito de la planificación escolar, sorprendentemente la participación espontánea va disminuyendo en la medida que aumenta la edad y se acerca la adolescencia (Boreham y Riddoch, 2001).

Dado el rol y la importancia en el ciclo vital de la persona, el ambiente escolar ha sido considerado el principal lugar de socialización del deporte para estudiantes de 12 a 15 años (Bryant, y McElroy, 1997; McPherson y Brown, 1988; Tuero del Prado, Zapico y González, 2012). Encontrándose experiencias con el deporte escolar y la educación física, donde estos contextos socio-educativos, han desempeñado un influyente rol en las decisiones actuales y futuras de los niños a participar en actividades deportivas (Curtis, McTeer y White, 1999; Tuero del Prado et al, 2012y White y Coakley, 1986). Weiss y Petlichkoff (1989) han informado que la tradición y trayectoria de la escuela, determina el énfasis y nivel de competitividad, la presión que se ejerce y las expectativas en torno al deporte. Bryant y MacElroy (1997) evidencia-

Fecha recepción: 20-11-14- Fecha envío revisores: 20-11-14- Fecha de aceptación: 07-07-15 Paula Francisca Ortiz Marholz portizmarholz@yahoo.com ron que el lugar donde existía mayor participación en deporte era el contexto escolar. Estos datos sugieren que la identidad deportiva de los jóvenes podría desarrollarse durante la vida escolar. Por ejemplo, el compromiso con el deporte del atleta-estudiante, ha sido identificado como un subproducto de la participación en el deporte escolar. A su vez, el disfrute, el cual es considerado como el mayor predictor de compromiso, combina la preferencia por la actividad, la satisfacción y el sentimiento de competencia en la práctica (García Mas et al., 2010).

Se ha planteado que uno de los elementos a considerar en el mantenimiento de la práctica es el compromiso deportivo, el cual ha sido trabajado como un buen elemento de prevención de abandono y asociado a la persistencia en el deporte (Cechini, González y Montero, 2007; Sousa, Torregrosa, Viladrich, Villamaría y Cruz, 2007). Cuando el compromiso deportivo es alto, existe una mayor sensación de satisfacción cuando se practica la actividad y por ende se entiende que el sentirse satisfecho y/o a gusto, disminuye la probabilidad de abandonarla (Sousa et al., 2007). En este sentido Scanlan, Russell, Magyary Scanlan (2009) plantean que la persistencia es una consecuencia conductual del compromiso deportivo y éste es entendido como la disposición psicológica de una persona que representa «el deseo y la decisión de seguir practicando el deporte» (Scanlan, Carpenter, Schmidt, Simons, y Keeler, 1993). El compromiso de un deportista hacia su actividad viene determinado por el grado de diversión que obtiene de la participación deportiva, por las inversiones personales, las oportunidades de implicación, las alternativas de implicación y la coacción o apoyo social (Scanlan, Simons, Carpenter, Schmidt y Keeler 1993; Scanlan, Russel, Beals y Scanlan, 2003). Con respecto a estudios confirmatorios del modelo, se ha encontrado que en población española las inversiones personales y las oportunidades de implicación mostraron baja confiabilidad, también se evidenció que coacción social se asocia negativamente al compromiso (Sousa et al., 2007). A su vez, según resultados encontrados por Sousa (2008), la variable alternativas de implicación (que predice negativamente el compromiso) en conjunto con diversión, generaron mayor explicación de la variancia del compromiso deportivo. En relación con el factor alternativas de implicación, éste se ha ido ampliando del modelo original, considerando que el deportista no solamente se puede sentir atraído a otras actividades, sino también existen otras prioridades que pueden ejercer presión en su vida cotidiana, como por ejemplo: estudios o familia (Scanlan et al., 2003).

Dado los antecedentes anteriores, se puede especular que el clima que en la escuela se genera en torno al deporte, podría contribuir/perjudicar al desarrollo del compromiso en sus alumnos. Sin dejar de conside- 
rar el importante rol que el entrenador ocupa (Cervelló, Escartí, y Guzmán, 2007; Sousa et al. 2007; García Calvo, Leo, Martín, y Sánchez Miguel, 2008), poco se sabe, en el contexto chileno, sobre la variables socioeducativa y su posible influencia sobre el compromiso con la actividad.

A la luz de los antecedentes descritos y dada la importancia de disminuir la realidad sedentaria y sus consecuencias en la salud física y mental, este estudio tiene como primer objetivo, relacionar el contexto socioeducativo (diferenciando tipos de establecimiento según su financiamiento) con las variables que componen el compromiso deportivo y revelar así, nueva información sobre estas variables en el contexto escolar y deportivo de iniciación. Como segundo objetivo, establecer diferencias entre alumnos y futbolistas que expresaron realizar otras actividades con los distintos factores del compromiso deportivo.

\section{Metodología}

\section{Participantes}

En este estudio participaron 930 varones con edades comprendidas entre los 10 y los 14 años $(M=12.2 ; D T=1.3)$. Se consideró como criterio de inclusión a todos los participantes entre las edades señaladas, que fueran parte de talleres o programas de fútbol en sus respectivos colegios o clubes. Como criterio de exclusión fue la permanencia menor a 3 meses en ese equipo o taller de fútbol, y/o que no hayan jugado, al menos 1 partido con su entrenador actual. La distribución por tipo de establecimiento ha sido de un $37.8 \%(n=352)$ pertenecientes a colegios públicos, 36.5\% $(n=340)$ pertenecientes a colegios particulares subvencionados, $14.4 \%(n=134)$ pertenecientes a colegios privados y $11.2 \%(n=104)$ pertenecientes a escuelas de fútbol (cadetes).

\section{Instrumentosy propiedades psicométricas}

El nivel de compromiso se evaluó a través de la versión española (Sousa, Torregrosa, Viladrich, Villamarín, y Cruz (2007) del Sport Commitment Model Questionnaire (SCQ, Scanlan, 1993), el SCQ-e. Este instrumento mide 6 factores influyentes en el nivel de compromiso deportivo los que se expresan a continuación con sus respectivos alpha de cronbach dados para esta investigación: Grado de Diversión (4 ítemes á = .77); Alternativas de Implicación (4ítemes á = .59); Inversiones Personales (3 ítemes á = .44); Oportunidades de Implicación (4 ítemes á = .67); Coacciones Sociales ( 7 ítemes á = .82) y Compromiso Deportivo (6 ítemes á = .73). Las respuestas fueron dadas a través de una escala tipo likert con un rango de 1 a 5 expresando nivel de acuerdo o desacuerdo con afirmaciones que mide cada uno de los factores, como «Me divierto realizando deporte esta temporada»; «Me gustaría hacer otras actividades en lugar de practicar deporte»; «Dedico mucho tiempo al deporte esta temporada»; «Si abandonase el deporte echaría de menos a mi entrenador»; «Hago deporte para agradar a mis amigos» $\mathrm{y}$ «Me siento orgulloso de decir a los demás que practico deporte».

La subescala de Inversiones personales no se incluyó en el estudio, dada su consistencia interna. Alternativas de Implicación se considera con las precauciones de uníndice en el límite de lo aceptable.Al igual que Oportunidades de Implicación, aunque presenta mejor índice.

Para caracterizar a la población y evaluar si en la práctica realizan otras actividades, se generó un cuestionario sociodemográfico en el cual se recogieron datos de los deportistas como edad, años de experiencia y frecuencia en la práctica deportiva de fútbol y la realización o no de otras actividades fuera del deporte estudiado.

\section{Procedimiento}

Se realizó un estudio transversal, desarrollándose un protocolo de actuación para llevar a cabo la recogida de datos en clubes y otro protocolo para establecimientos educacionales, previamente aprobada por el comité de ética de la Universidad de Playa Ancha de Valparaíso, respetando también los principios éticos de la declaración de Helsinski

Una vez cumplido con el consentimiento informado, se coordinó lugar, fecha y hora de aplicación. Se informó a los deportistas de que su participación era voluntaria y las respuestas serían tratadas confiden- cialmente. Se realizó la aplicación en el vestuario (en el caso de los clubes) y en salas de clases en caso de los establecimientos educacionales, sin la presencia del entrenador. El proceso de administración de los cuestionarios duró aproximadamente 25 minutos. El investigador principal estuvo presente en el momento en que los sujetos completaron los cuestionarios, e insistió en la posibilidad de preguntar cualquier tipo de duda que apareciese durante el proceso.

\section{Análisis estadístico de los datos}

Para obtener los resultados se ha realizado análisis descriptivo, el análisis de fiabilidad y análisis de varianza intersujetos, donde se han estudiado los efectos simples y los de interacción. Para el análisis de los datos se utilizó SPSS 15.0.

Para comprobar la consistencia interna de los diferentes cuestionarios, se realizó un análisis factorial exploratorio con los diferentes ítems que forman parte de cada instrumento, obteniendo una estructura adecuadadecadaunadelas escalas, tras el análisis denormalidad(KolmogorovSmirnov) (Tabla 1).

Con el objetivo de comprobar las diferencias existentes entre los tipos de establecimiento y entre los que expresan realizar o no otras actividades independientes del fútbol, se decidió llevar a cabo la aplicación de prueba de los efectos intersujetos y en caso de encontrar diferencias, realizar análisis de comparaciones múltiples Bonferroni.

A cada variable dependiente (6 escalas que componen el constructo de compromiso deportivo), se le realizó análisis de varianza en razón del tipo de establecimiento, la realización de otras actividades y la relación entre ambas variables independientes. A continuación se exponen los resultados de las diferencias significativas encontradas

\section{Resultados}

En la Tabla 1 se muestran los valores descriptivos, de normalidad, asimetría y curtosis de los datos.

Como indican los resultados, la muestra se distribuye con normalidad y los valores obtenidos mostraron que los participantes lo pasaban bien en su actividad a través del grado de diversión, sentían que se les dan oportunidades para participar y mostraban compromiso deportivo general. Se observó bajo grado de presión, es decir, el grado de coacción social no se percibió de manera negativa. Lo mismo sucede con alternativas de implicación, es decir percibieron que existen otras actividades que pudieran interesarle o a las que les dedican tiempo, pero no son altamentellamativas.

Para poder identificar las diferencias entre variables modulares y compromiso deportivo, se realizó, en primer lugar, un análisis de varianza bifactorial y así conocer si las variables independientes eran significativas. En la Tabla 2 se puede observar que se ha encontrado significancia en el efecto de la variable tipo de centro con todas las variables del compromiso deportivo. También se encontró efecto significativo de otras actividades sólo con alternativas de implicación y por último se observa efecto significativo de la interacción de tipo de centro con otras actividades sobre compromiso deportivo.

Con respecto a la primera variable del SCQ-e, diversión, se observa en la Tabla 3 que los alumnos pertenecientes al establecimiento municipalizado muestran diferencia significativa de las medias observadas a

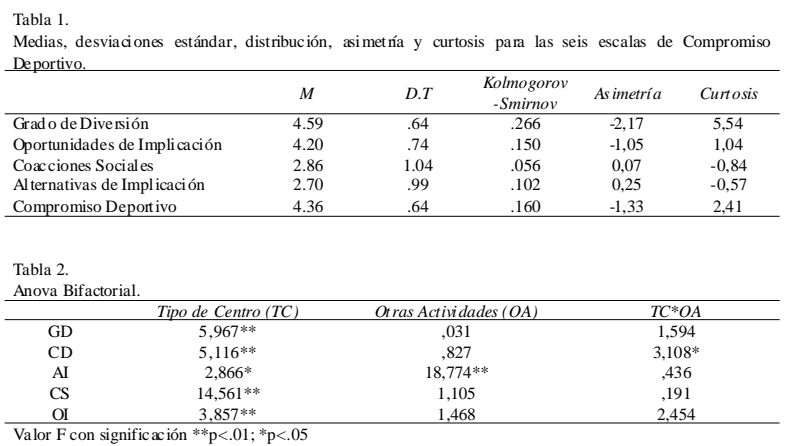


nivel de .05 y de menor grado que los alumnos de los centros particular subvencionado, particular pagado y escuelas de fútbol.

En la Tabla 4 se indica que la segunda variable evaluada en el SCQe, compromiso deportivo, muestra diferencias en el tipo de centro municipalizado con particular subvencionado y con escuela de fútbol. El primero se muestra con menor compromiso de manera significativa en relación con el otro centro educacional y la escuela de fútbol.

En relación con la tercera variable en estudio, alternativas de implicación, en la Tabla 5 se observa que existe diferencia entre los tipos de centro municipal y escuelas de fútbol. Siendo el primero quien muestra mayor puntuación en esta variable, lo que dice que los alumnos de los centros municipales evalúan en mayor medida participar en otras actividades que los pertenecientes a las escuelas de fútbol. En esta línea también se encontró diferencia entre los particulares pagados con los municipales, en donde los primeros muestran menor grado de alternativas de implicación con respecto a los municipales.

Con respecto a coacciones sociales, se pudo establecer diferencias significativas en los tres tipos de centros con el municipalizado. Los alumnos de los municipales muestran mayor percepción de presión social que los pertenecientes a los centros particular subvencionado, particular pagado y escuelas de fútbol. En esta línea, los alumnos pertenecientes a centros particular subvencionado y particular pagado muestran menor percepción de presión social que los pertenecientes a los municipales. Esto queda expuesto en la Tabla 6.

En la tabla 7 se observa que la sexta variable, Oportunidades de Implicación, muestra diferencias en el tipo de centro, donde los alumnos municipales perciben menos oportunidades de implicación que los alumnos pertenecientes a las escuelas de fútbol y los alumnos de los particulares pagados muestran mayor percepción de oportunidades para participar que los pertenecientes a los establecimientos municipales.

En referencia con el efecto encontrado entre otras actividades y

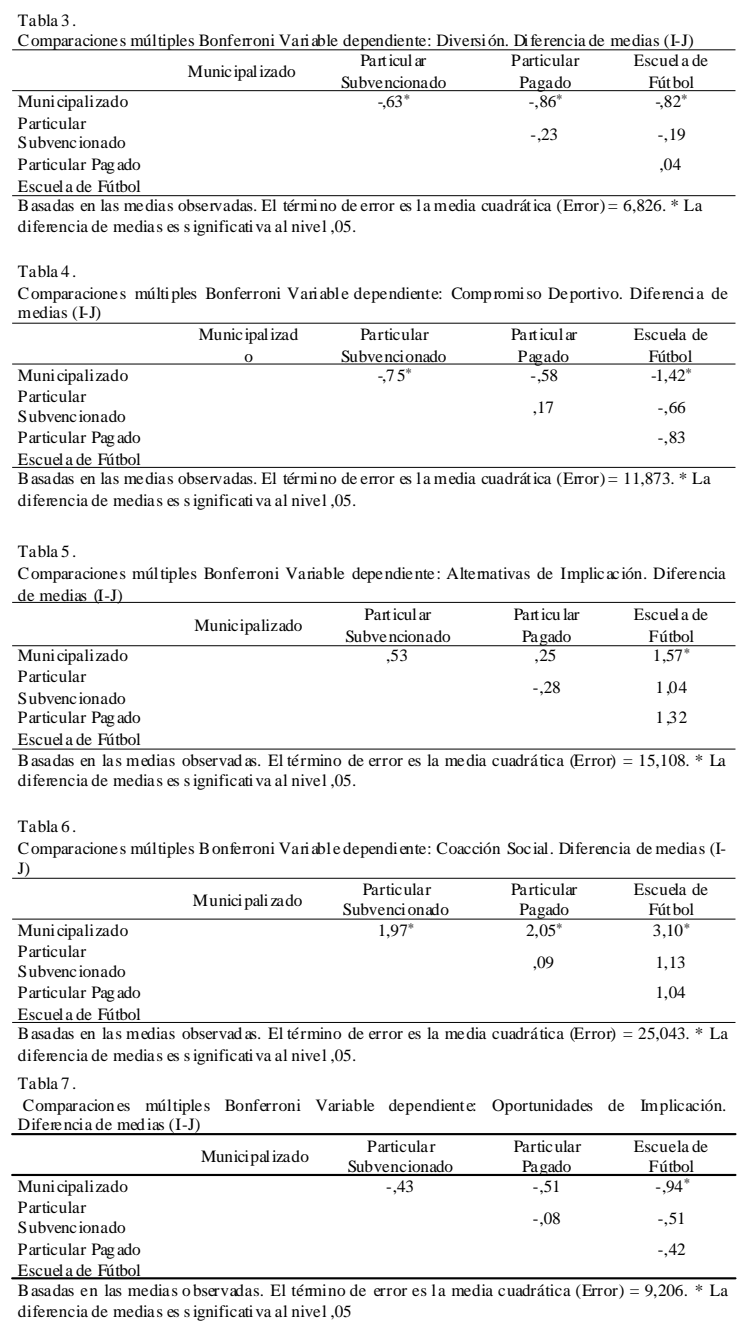

Tabla 8 \begin{tabular}{cc} 
Di ferencias de medias entre los que realizan otras actividades y los que no las realizan. \\
\hline Otras Actividades & Media \\
\hline Si & 11,218 \\
No & 9,871 \\
\hline
\end{tabular}

Tabla 9

Comparaciones por pares Variable dependiente: Compromiso Deportivo

\begin{tabular}{llll}
$\begin{array}{l}\text { Realiza otras } \\
\text { actividades: SI }\end{array}$ & Municipalizado & $\begin{array}{l}\text { Particular } \\
\text { Subvencionado }\end{array}$ & $\begin{array}{l}\text { Partic } \\
\text { Pagado }\end{array}$ \\
\hline
\end{tabular} \begin{tabular}{lrrr} 
& Subvencionado & Pagado & \multicolumn{1}{c}{ Fútbol } \\
\hline Munic ipal izado & $-1,024^{*}$ &,- 044 & $-1,388$
\end{tabular} Subvencion

Particul ar Pagado

Escuela de Fútbo

Realiza otras $\quad$ Particular $\quad$ Particular Escuela de
\begin{tabular}{lcccc} 
actividades: NO & Municipalizado & $\begin{array}{l}\text { Particular } \\
\text { Subvencionado }\end{array}$ & Pagado & Fútbol \\
\hline &,- 356 & $-1,614^{*}$ & \\
\hline
\end{tabular} $\begin{array}{llll}\text { Municipal izado } & -, 356 & -1,614^{*} & -1,422^{*} \\ \text { Particular } & & -1,258 & -1,066\end{array}$

Particular Pagado

1,066

, 193

.

Ajuste para comparaciones múltiples: Bonferroni

alternativas de implicación en la población total, se puede observar que los que expresaron realizar otras actividades mostraron mayor nivel de atracción o perciben otras actividades como potencialmente más atractivas.

Finalmente, con respecto a la relación entre tipo de establecimiento y la realización de otras actividades los alumnos pertenecientes a los centros municipales, muestran menor compromiso deportivo que los que realizan otras actividades de los centros particulares subvencionados. Por otra parte, dentro de los que no realizan otras actividades, nuevamente los alumnos de los establecimientos municipales muestran menor grado de compromiso deportivo que los pertenecientes a establecimientos particulares pagados y escuelas de fútbol.

\section{Discusión}

En relación con el primer objetivo de este estudio, buscar diferencias en el nivel de compromiso deportivo en función de variables socioeducativas como los tipos de establecimientos de acuerdo a su financiamiento, se evidenció que el establecimiento municipalizado presentó diferencias de mayor puntuación en las variables que pueden actuar como negativas para el compromiso, como alternativas de implicación y coacciones sociales. En contraparte, menor puntuación en aquellas que según el modelo actúan como propulsoras del compromiso: diversión, compromiso deportivo y oportunidades de implicación.

Si bien, la mayoría de los estudios sobre compromiso han estudiado las influencias de los otros significativos sobre este y en definitiva sobre los procesos individuales que hacen desarrollar mayor o menor compromiso (García Calvo et al., 2008; Torregrosa, Viladrich, Villamarín y Cruz, 2008; Almagro, Sáenz-López, González-Cutre y Moreno-Murcia, 2011; Leo et al., 2011; Saybani, Yusof, Hassan y Zardoshtian, 2013; Santi, Bruton, Pietrantoni y Mellalieu, 2014, Ortiz, Chirosa, Martín, Reigal y Mass, 2014), en este estudio se genera nueva información acerca del contexto socio educativo, en donde se puede especular acerca de la realidad pública versus la semipública, privada o de escuelas de fútbol. Coincidiendo con Carreres-Ponsoda, Escartí, Cortell-Tormo, Fuster-Lloret y Andreu-Cabrera (2012), donde se plantea que la alta calidad y la estructura tanto de los programas escolares como fuera de ese contexto, tienen el potencial para apoyar y promover el desarrollo de los jóvenes. A su vez, también es importante considerar las conductas de los profesionales a cargo de estos programas, tal como lo plantea Sousa, Smith y Cruz (2008), donde se halló que aquellos entrenadores que utilizan más conductas reforzadoras, disminuían conductas punitivas. También que los deportistas consideran apropiado ser criticado tras cometer errores, pero parece que valoran, sobre todo, conductas de apoyo que ayudan a crear un ambiente interpersonal positivo.

Estas conductas reforzadoras de los entrenadores tenían relación con el disfrute y el compromiso. A su vez Côté, Strachan and FraserThomas (2008), postulan que la necesidad de que los niños y adolescentes tengan su foco en el rendimiento del partido puede ser disuasivo para la mejora de las habilidades individuales o de la táctica del equipo. Este foco puede afectar negativamente en las decisiones de los entrenadores y de acuerdo a los hallazgos de este estudio, esto puede venir influenciado por el contexto socioeducativo. El deporte es un contexto 
en el cual se pueden desarrollar altos niveles de desarrollo positivo en niños y jóvenes (Zarret et al., 2009 y Carreres-Ponsoda et al., 2012), sin embargo es importante considerar los contextos sociales, educativos y familiares que van permitiendo el desarrollo de conductas que ayudan a mantenerse y comprometerse en la actividad (Jones, Dunn, Holt, Sullivan y Bloom, 2009; Tuero del Prado et al. 2012). Y si el contexto deportivo es organizado, estructurado y supervisado genera resultados positivos en sus participantes (Whitt-Glover et al., 2009; Zarret et al., 2009; González, Manrique, López, 2012).

En este sentido, se puede plantear que los tipos de establecimientos públicos estarían respondiendo de menor manera frente los factores protectores ante el abandono. Sin embargo no es el contexto en sí el determinante, es decir, no por el hecho de pertenecer a un contexto socioeducativo en particular, se tenderá a presentar mayor o menor nivel de compromiso deportivo. Se evidencia que ante un contexto de relaciones complejas, es importante cuestionarse sobre la calidad de los programas deportivos, el perfil del profesional a cargo y los objetivos que persigue el establecimiento educacional; de esta manera poder relacionar con las presiones que se puedan estar ejerciendo en sus alumnos, concordando con Weiss y Petlichkoff (1989), Sousa et al. (2008) y Reverter, Plaza, Jové y Mayolas (2012).

Con respecto a la segunda variable estudiada, diferencias entre alumnos que expresaron realizar otras actividades con los distintos factores del compromiso deportivo, se encontraron resultados en 2 variables que componen este modelo. Por una parte, se pudo comprobar diferencias de la población total en la variable alternativas de implicación (sin diferenciar por nivel socioeducativo), donde los que expresaron realizar otras actividades de manera constante presentaron mayor nivel de atracción o perciben otras actividades alternativas como potencialmente más atractivas en comparación con el fútbol, en este caso. Es decir, los jugadores pueden atribuir, potencialmente, grados de importancia a otras actividades en lugar de jugar fútbol. Esto plantea un nuevo análisis en referencia a lo planteado por Scanlan et al.(1993), donde, en la primera validación del modelo, consideraron como factible compaginar dos actividades sin necesidad de escoger una de ellas, sugiriendo que la variable alternativa de implicación era difícil de estudiar en ciertas edades.

Sousa (2008), encontró que jugadores de fútbol de clubes de Cataluña entre 14 y 16 años, atribuían poca importancia a otras actividades que podían realizar en lugar de jugar fútbol. En nuestro estudio, la media en esta variable es baja, al igual que el estudio de Sousa, sin embargo, se añade que existe diferencia entre los que realizan otras actividades y los que no, influyendo potencialmente en el compromiso y en la adherencia en la actividad el hecho de estar participando en otras actividades.

Por otra parte, se encontró diferencias por nivel socioeducativo entre los que sí participaban en otras actividades y los que no, en la variable compromiso deportivo. Dentro de las comparaciones entre los que sí realizaban otras actividades, los alumnos de establecimientos públicos mostraron menor compromiso deportivo que los que también realizaban otras actividades pero de los establecimientos particular subvencionado. A su vez, de los alumnos que expresaron no realizar otras actividades, se encontró diferencias en donde los alumnos de los colegios públicos mostraron menor compromiso deportivo que sus pares de los colegios pagados y escuelas de fútbol.Al no encontrarse evidencia acerca de estudios que hayan evaluado el compromiso deportivo considerando el contexto socioeducativo, estos hallazgos no pueden ser comparados, sin embargo podemos coincidir parcialmente en razón de resultados como los de Sousa (2008) donde la variable alternativas de implicación se relaciona negativamente con el compromiso y con diversión.

Si bien es cierto que la adolescencia se caracteriza por la búsqueda e intereses múltiples, y por tal razón puede darse la participación en distintas actividades, concordamos con Carreres-Ponsoda et al. (2012) que plantean que la diversidad individual de los jóvenes es el resultado de cambios en las relaciones complejas que un individuo mantiene con su contexto.

En la relación tipo de establecimiento con realización de otras acti- vidades, se logró determinar que, al igual que la tendencia en los resultados de la evaluación del compromiso deportivo, los alumnos pertenecientes a escuelas municipales y que realizaban otras actividades mostraron menor compromiso deportivo que sus pares de los establecimientos particulares subvencionados. En esta línea, los que no realizaban otras actividades de manera regular también mostraron menor compromiso deportivo que sus iguales de escuelas particulares pagadas y escuelas de fútbol. Una de las características del compromiso deportivo es la persistencia y estudios como los de Gonçalves, Coelho, Carvalho y Gonçalves (2011) proponen que la voluntad para alcanzar la excelencia en el rendimiento puede ser considerado como una condición para la participación más especializada y prácticas más exigentes, concordando así con la definición de alternativas de implicación del modelo de compromiso deportivo, donde la realización de otras actividades genera menor compromiso al tener de dedicar tiempo y energía a ellas en desmedro de, en este caso, el fútbol. Tiempo y energía que pudiese influir en los niveles de compromiso y excelencia.

Como limitante de esta investigación es que a pesar del número elevado de participantes, el fútbol fue el único deporte estudiado y esto puede limitar las inferencias para el deporte en general. Una segunda limitante, sería la complementación de la percepción de compromiso deportivo con datos como porcentajes de asistencia a entrenamiento y partidos, inversiones personales concretas, etc.

\section{Conclusiones}

Existen diferencias entre establecimientos de distinto contexto socioeducativo, siendo los públicos o municipales los que presentan menores índices en las variables que potencian el compromiso (Diversión, Compromiso Deportivo y Oportunidades de Implicación) y mayores índices en los factores que predicen negativamente el compromiso (Alternativas de Implicación y Coacciones sociales).

Se puede plantear, según los resultados, que el realizar diversas actividades afecta el nivel de compromiso y la adherencia, por lo que el deporte debiera centrarse en la diversión, proveer la oportunidad de participar, de mejorar habilidades, obtener una mejor comprensión de sí mismos y de establecer relaciones interpersonales prolongadas. Obteniendo así, un efecto positivo en los niños de la escuela evitando el abandono y propiciando, de esta manera, una población más activa físicamente, sobre todo en los contextos menos favorecidos económicamente.

Se contribuye con nueva información acerca de los contextos socioeducativos, poniendo la mirada en las expectativas, procesos de selección del personal y construcción de los programas, centrándose en la calidad para desarrollar actividades deportivas que ofrezcan equilibrio y profundidad de diversas experiencias educativas de los estudiantes, así como habilidades positivas como la persistencia.

Como proyecciones a futuro, próximas investigaciones pueden enfocarse precisamente en indagar en las presiones que se ejercen y que contribuyen o afectan a las conductas de los entrenadores y éstas a la percepción de disfrute de los practicantes. Analizando los porcentajes de participación a principio de la temporada y al final.

A su vez, se podrían investigar diferencias entre los programas impartidos en los distintos tipos de establecimiento, ya sea en su frecuencia, infraestructura y objetivos a conseguir.

Finalmente, ampliar la muestra tanto a deportes individuales como colectivos y a género masculino y femenino.

\section{Agradecimientos}

Se agradece a la Universidad de Playa Ancha de Valparaíso, Chile, ya que esta investigación fue financiada por fondos concursables de dichainstitución.

\section{Referencias}

Almagro, B. Sáenz-López, P. González-Cutre, D., y Moreno-Murcia, J. (2011). Clima motivacional percibido, necesidades psicológicas y motivación intrínseca como predictores del compromiso deportivo 
en adolescentes. Revista Internacional de Ciencias del Deporte, 7(7), 250-265.

Anderson, D., y Cychosz, C. (1995). Exploration of the relationship between exercise behavior and exercise identity. Journal of Sport Behavior, 18(3), 159-167.

Boreham C., y Riddoch, C. (2001). The physical activity, fitness and health of children. Journal of Sport Sciences, 19 (12), 915-929.

Brettschneider, W. D., y Heim, R. (1997). Identity, sport and youth development. In, Fox, K.R. (ed.), The physical self: from motivation to well-being, Champaign, Ill., Human Kinetics Publishers, 205227.

Bryant, J., y McElroy, M. (1997). Sociological Dynamics of Sport an Exercise. Colorado: Morton Publishing Company.

Cachán, R. (2011). El balonmano y los procesos de construcción de identidad en León. Revista de Ciencias del Deporte, 7 (2), 79-90

Carreres-Ponsoda, F., Escartí, A., Cortell-Tormo, J., Fuster-Lloret, V., y Andreu-Cabrera, E. (2012). The relationship between out-of-school sport participation and positive youth development. Journal of Human Sport \& Exercise, 7(3), 671-682.

Cechini, J. A., González, C., y Montero, J. (2007). Participación en el deporte y fair play. Psicothema, 19, 57-64.

Cervelló, E. M., Escarti, A., y Guzmán, J. F. (2007). Youth sport dropout from the achievement goal theory. Psicothema, 19, 65-71.

Côté, J., Strachan, L., y Fraser-Thomas, J. (2008). Participation, personal development and performance through youth sport. In N. L. Holt (Ed.), Positive youth development through sport, London: Routledge, 34-40.

Curry, T. (1993). The effects of receiving a college letter on the sport identity. Sociology of Sport Journal, 10, 73-87.

Curry, T., y Weaner J. (1987). Sport identity salience, commitment and the involvement of self in role: measurement issues. Sociology of Sport Journal, 4, 280-288.

Curtis, J., McTeer, W., y White, P. (1999). Exploring effects of school sport experiences on sport participation in later life. Sociology of Sport Journal, 16(4), 348-365.

García Calvo, T., Leo, F. M., Martín, E., y Sánchez Miguel, P. A. (2008). El compromiso deportivo y su relación con factores disposicionales y situacionales contextuales de la motivación. Revista Internacional de Ciencias del Deporte, 12(4), 45-58.

García Ferrando, M. (2006). Veinticinco años de análisis del comportamiento deportivo de la población española (1980-2005). Revista internacional de sociología, 44, 15-38.

García Más, A., Palou, P., Gili, M., Ponseti, X., Borras. P., Vidal, J., Cruz, J.,... y Sousa, C. (2010). Commitment, Enjoyment and Motivation in Young Soccer Competitive Players. The Spanish Journal of Psychology, 13, 609-616

Gonçalves, C., Coelho, M., Carvalho, H., y Gonçalves, Â. (2011). Why do they engage in such hard programs? The search for excellence in youth basketball. Journal of Sport Science and Medicine, 10, 458464.

González, M., Manrique, J., López, V. (2012). Valoración del primer curso de implantación de un programa municipal integral de deporte escolar. Retos. Nuevas tendencias en Educación Física, Deporte y Recreación, 21, 14-18.

Greendorfer S. (2002). Socialization processes and sport behavior. In: Horne TS., editor. Advances in sport Psychology. Champaign, IL: Human Kinetics, 377- 401.

Jones, M., Dunn, J., Holt, N., Sullivan, P., y Bloom, G. (2009). Exploring the «5Cs» of positive youth development in sport. Journal of Sport Behavior, 34 (3), 250-267.

Lau, P., Fox, K., y Cheung M. (2004). Psychosocial and Socioenvironmental correlates of sport identity and Sport participation in secondary school-age children. European Journal of Sport Science, 4, 1-21. DOI: $10.1080 / 17461390400074301$

Leo, F., Sánchez, P., Sánchez, D., Alonso, D., y García Calvo, T. (2011). Análisis de la incidencia del clima motivacional y la cohesión de equipo sobre el grado de compromiso de jóvenes futbolistas. Revista Cultura, Ciencia y Deporte, 16 (6), 5-13.

McPherson, B., y Brown, B. (1988). The structure, processes and consequences of sport for children. In: Smoll F., editor. Children in sport. 3rd ed. Champaign, IL: Human Kinetics.

Ortiz, P., Chirosa, L., Martín, I., Reigal R.E., y García- Mas, A. (2014) Predicción del Compromiso Deportivo a través de la percepción de Clima Motivacional de madre, padre y entrenador, en futbolistas jóvenes. Artículo presentado para publicación.
Reverter, J., Plaza, D., Jové, M., Mayolas, M. (2012). Influencia de los técnicos en el deporte extraescolar: El caso de la ciudad de Torrevieja. Retos. Nuevas tendencias en Educación Física, Deportes y Recreación, 22, 76-80.

Roulin, N., y Bangerter, A. (2013). Extracurricular activities in young applicants' résumés: What are the motives behind their involvement? International Journal of Psychology 48 (5), 871-880.

Santi, G., Bruton, A., Pietrantoni, L., y Mellalieu, S. (2014). Sport commitment and participation in master swimmers: The influence of coach and teammantes. European Journal of Sport Science. DOI:10.1080/17461391.2014.915990.

Saybani, H., Yusof, A., Hassan, A., y Zardoshtian, S. (2013). Athlete's Satisfaction as Mediator of Transformational Leadership Behaviors of Coaches and Football Players'Sport Commitment Relationship. World Applied Sciences Journal, 21 (10), 1475-1483.

Scanlan, T. K., Simons, J. P., Carpenter, P. J., Schmidt, G. W., y Keeler, B. (1993). The sport commitment model: Measurement development for the youth-sport domain. Journal of Sport and Exercise Psychology, 15, 16-38.

Scanlan, T. K., Russell, D. G., Magyar T. M., y Scanlan L. A. (2009) Project on elite athlete commitment (PEAK). III. An examination of external validity across gender and the expansion and clarification of the sport commitment model. Journal of sport and exercise Psychology, 31, 685 - 705.

Scanlan, T.K., Russell, D.G Beals, N.C., y Scanlan, L.A. (2003). Project on elite athlete commitment (PEAK): I. Introduction and methodology. Journal of Sport and Exercise Psychology, 25, 360376.

Scanlan, T.K., Carpenter, P., Schmidt, G.W., Simons, J.Y., y Keeler, B. (1993). An introduction to the Sport Commitment Model. Journal of Sport and Exercise Psychology, 15, 1-15.

Sousa, C. (2008). Efectos del Programa de Asesoramiento Personalizado a Entrenadores (PAPE) en la conducta del entrenador y en el compromiso deportivo. (Tesis Doctoral, Universidad de Barcelona, España). Extraída de http://ddd.uab.cat/record/37939

Sousa, C., Torregrosa, M., Viladrich, C., Villamarín F., y Cruz, J. (2007). The commitment of young soccer players. Psicothema, 19, 256262.

Sousa, C., Smith, R., y Cruz, J. (2008). An Individualized Behavioral GoalSetting Program for Coaches. Journal of Clinical Sport Psychology, 2, 258-277.

Sousa, C., Torregrosa, M., Viladrich, C., Villamarín F., y Cruz, J. (2007). The commitment of young soccer players. Psicothema, 19, 256262.

Theodorakis, Y. (1994). Planned behavior, attitude strength, role identity and the prediction of exercise behavior. The Sport Psychologist, 8, 149-165.

Torregrosa, M., Sousa, C., Viladrich, C., Villamarín, F., y Cruz, J. (2008). El clima motivacional y el estilo de comunicación del entrenador como predictores del compromiso en futbolistas jóvenes. Psicothema, 20 (2), 254-259.

Tuero del Prado, C., Zapico, B., y González, R. (2012). Deporte en edad escolar y agentes sociales: estudio preliminar sobre la relación entre familia, monitores y deportistas en provincia de León. Retos. Nuevas tendencias en Educación Física, Deporte y Recreación, 21, 34-37

Universidad de Concepción, Encuesta Nacional de Hábitos de Actividad Física y Deportes en la población chilena de 18 años y más (2012). Reportado por Instituto Nacional de Deportes, Chile. Extraída de http://www.ind.cl/estudios-e-investigacion/encuesta-actividad-fisica2012/Paginas/encuesta-2012.aspx

Weiss, M. R., y Petlichkoff, L.M. (1989). Children's motivation for participation in and withdrawal from sport: Identifying the missing links. Pediatric Exercise Science, 1, 195-211.

White, A., y Coakley, J. (1986). Making decisions: The response of young people in the Medway Towns to the «Ever thought about sport?» Campaign, London: West Sussex Institute of Higher Education.

Whitt-Glover, M., Taylor, W., Floyd, M., Yore, M., Yancy, A., y Matthews, C. (2009). Disparities in psysical activity and sedentary behavooir among US children and adolscents: prevalence, correlates and intervention implications. Journal of Public Health Policy, 30, 309334. DOI: 10.1057/jphp.2008.46.

Zarret, N., Fay, K., Li, Y., Carrano, J., Phelps, E., y Lerner, R. (2009). More tan child's play: Variable-and pattern-centered approaches for examining effects of sports participation on youth development. Developmental Psychology, 45 (2), 368-382. 